\title{
Comparison of Superficial Surgical Site Infection Between Delayed Primary Versus Primary Wound Closure in Complicated Appendicitis
}

\author{
A Randomized Controlled Trial
}

\author{
Boonying Siribumrungwong, MD, ${ }^{*} \dagger$ Anuwat Chantip, MD, $\ddagger$ Pinit Noorit, MD, $\S$ Chumpon Wilasrusmee, MD, $\uparrow$ \\ Winai Ungpinitpong, MD, || Pradya Chotiya, MD, ${ }^{* *}$ Borwornsom Leerapan, MD, PhD, $\dagger \dagger$

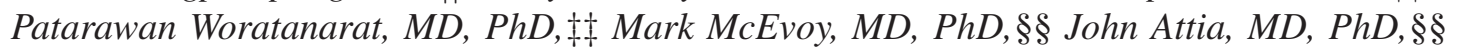 \\ and Ammarin Thakkinstian, $P h D^{*}$
}

\begin{abstract}
Objective: To compare superficial surgical site infection (SSI) rates between delayed primary wound closure (DPC) and primary wound closure (PC) for complicated appendicitis.

Background: SSI is common in appendectomy for complicated appendicitis. DPC is preferentially used over PC, but its efficacy is still controversial.

Methods: A multicenter randomized controlled trial was conducted in 6 hospitals in Thailand, enrolling patients with gangrenous and ruptured appendicitis. Patients were randomized to PC (ie, immediately wound closure) or DPC (ie, wound closure at postoperative days 3-5). Superficial SSI was defined by the Center for Disease Control criteria. Secondary outcomes included postoperative pain, length of stay, recovery time, quality of life, and cost of treatment. Results: In all, 303 and 304 patients were randomized to PC and DPC groups, and 5 and 4 patients were lost to follow-up, respectively, leaving 300 and 298 patients in the modified intention-to-treat analysis. The superficial SSI rate was lower in the PC than DPC groups [ie, 7.3\% (95\% confidence interval 4.4, $10.3)$ vs $10 \%(95 \%$ CI $6.6,13.3)$ ] with a risk difference (RD) of $-2.7 \%$ $(-7.1 \%, 1.9 \%)$, but this RD was not significant. Postoperative pain, length of stay, recovery times, and quality of life were nonsignificantly different with
\end{abstract}

From the *Section for Clinical Epidemiology and Biostatistics, Faculty of Medicine, Ramathibodi Hospital, Mahidol University, Bangkok, Thailand; $\dagger$ Department of Surgery, Faculty of Medicine, Thammasat University Hospital, Thammasat University, Pathumthani, Thailand; $\ddagger$ Department of Surgery, Lampang Regional Hospital, Lampang, Thailand; §Department of Surgery, Chonburi Hospital, Chonburi, Thailand; - Department of Surgery, Faculty of Medicine, Ramathibodi Hospital, Mahidol University, Bangkok, Thailand; ||Department of Surgery, Surin Hospital, Surin, Thailand; **Department of Surgery, Pathum Thani Hospital, Pathum Thani, Thailand; ††Department of Community Medicine, Faculty of Medicine, Ramathibodi Hospital, Mahidol University, Bangkok, Thailand; ł†̣Department of Orthopedics, Faculty of Medicine, Ramathibodi Hospital, Mahidol University, Bangkok, Thailand; and $\S \S C e n t r e$ for Clinical Epidemiology and Biostatistics, Hunter Medical Research Institute, School of Medicine and Public Health, University of Newcastle, Newcastle, NSW, Australia.

Funding: This study was grant by Health Systems Research Institute, Thailand (grant number 59-062).

The authors report no conflicts of interest.

Supplemental digital content is available for this article. Direct URL citations appear in the printed text and are provided in the HTML and PDF versions of this article on the journal's Web site (www.annalsofsurgery.com).

This is an open access article distributed under the terms of the Creative Commons Attribution-Non Commercial-No Derivatives License 4.0 (CCBY-NC-ND), where it is permissible to download and share the work provided it is properly cited. The work cannot be changed in any way or used commercially without permission from the journal.

Reprints: Ammarin Thakkinstian, $\mathrm{PhD}$, Section for Clinical Epidemiology and Biostatistics, Faculty of Medicine, Ramathibodi Hospital, Mahidol University, 270 Rama VI Road, Rachatevi, Bangkok 10400, Thailand.

E-mail: ammarin.tha@mahidol.ac.th.

Copyright (C) 2017 The Author(s). Published by Wolters Kluwer Health, Inc. ISSN: 0003-4932/17/26704-0631

DOI: $10.1097 /$ SLA.0000000000002464 corresponding RDs of $0.3(-2.5,3.0),-0.1(-0.5,0.3),-0.2(-0.8,0.4)$, and $0.02(-0.01,0.04)$, respectively. However, costs for PC were 2083 (1410, 2756) Baht cheaper than DPC ( $\$ 60$ USD).

Conclusions: Superficial SSI rates for the PC group were slightly lower than DPC group, but this did not reach statistical significance. Costs were significantly lower for the PC group.

Keywords: appendicitis, delayed primary closure, surgical site infection, wound closure, wound infection

(Ann Surg 2018;267:631-637)

$\Delta$ ppendicitis is a common surgical emergency. The rate of apA pendectomy in a Korean study was 14 per 10,000 population per year, of which $21 \%$ was for complicated appendicitis (ie, gangrenous and ruptured). ${ }^{1}$ Superficial surgical site infection (SSI) is a common complication (ie, 9\%-53\% ${ }^{2}$ ) in this condition as compared with simple appendicitis, ${ }^{3}$ and adversely affects both patients and the healthcare system. ${ }^{4}$

Delayed primary wound closure (DPC), first introduced in World War $\mathrm{I},{ }^{5}$ is an intervention which aims to decrease superficial SSI, ${ }^{5}$ by reducing bacteria and increasing blood supply ${ }^{6}$ and oxygen ${ }^{7}$ at the surgical site. Instead of suturing a wound immediately after operation [primary wound closure (PC)], the wound is left open and sutured on postoperative day 3 to $5 .^{8}$ This delay has been found to decrease SSI in other contaminated wounds., ${ }^{910}$ However, it is invasive, with dressing changes and re-suturing, and increases length of stay and cost of treatment. ${ }^{11}$

Given advances in antibiotics and perioperative care over recent years, ${ }^{12}$ we questioned whether DPC was still required as routine practice. A recent meta-analysis ${ }^{2}$ of 6 randomized controlled trials (RCTs, n $=234$ vs 182 for PC vs DPC) showed slightly lower superficial SSI rates in PC than DPC (ie, $23 \%$ vs $26 \%$ ). However, these included RCTs were of low quality and the pooled effect was imprecise. Although DPC is still debated, it is still the current standard of practice. ${ }^{13}$ Therefore, we conducted an RCT aiming to compare superficial SSI between DPC and PC groups after appendectomy with right lower-quadrant abdominal wound incision in complicated appendicitis. Secondary outcomes included recovery time, postoperative pain, length of stay (LOS), quality of life (QoL), and costs of treatment.

\section{METHODS}

A multicenter parallel RCT was conducted from November 2012 to February 2016, across 6 hospitals in Thailand, that is, 2 university hospitals (Thammasat and Ramathibodi hospitals), Pathum Thani (the Central region), Chonburi (the Eastern region), Surin (the North-Eastern region), and Lampang (the Northern 
region). This study was approved by the Ethics committee of Ramathibodi Hospital and the collaborating hospitals, and registered with ClinicalTrials.gov (ID NCT01659983).

\section{Participants}

Eligibility criteria were: age $\geq 18$ years, appendectomy with a right lower-quadrant abdominal incision, not pregnant, providing informed consent, and no history of the after diseases/conditions: obesity [body mass index (BMI) $\geq 40 \mathrm{~kg} / \mathrm{m}^{2}$ ], autoimmune diseases, end-stage renal/liver disease, or HIV. Intraoperative eligibility criteria were then assessed: erythematous or swollen appendix with a necrotic wall (dark, grayish color), or rupture, rupture during the procedure, or presence of frank pus. ${ }^{3}$

\section{Randomization}

Randomization was performed by permuted blocks of 4 to 6 , stratified by hospital with assignment provided by an independent statistician using STATA version 12.0. Treatment assignments were stored in sealed opaque envelopes, which were opened by scrub nurses intraoperatively, before closure of the appendectomy incision.

\section{Blinding}

Neither patients nor attending physicians were blinded to intervention due to obvious constraints. Superficial SSIs were assessed using standard criteria and monitoring procedures (see below) to minimize ascertainment bias. However, research assistants involved in subjective outcome assessments (ie, recovery period, pain, and QoL) were blinded.

\section{Interventions}

Appendectomy and wound closure were done by surgical staff or surgical residents under supervision. For PC, the wound was closed immediately after the operation using a nonabsorbable monofilament suture or stapler. Dry dressings were applied daily until stitches were removed. For DPC, the wound was left open with twice daily saline-soaked gauze, and closed on postoperative day 3 to 7 using the same suture as PC.

\section{Cointervention}

Cointerventions were standardized, including use of antibiotics, pain control, wound dressings, and closed suction drains. All patients were prescribed pre/postoperative intravenous antibiotics until body temperature was $<37.8^{\circ} \mathrm{C}$ for 24 to 48 hours, then switched to oral antibiotics for 7 to 10 days. Antibiotics were chosen to cover enteric gram-negative and facultative/anaerobic bacilli. ${ }^{14}$ Patients were prescribed intravenous opioids (ie, morphine $3-5 \mathrm{mg}$ or pethidine $25-$ $50 \mathrm{mg}$ ) as requested every 4 hours, switching to paracetamol or nonsteroidal anti-inflammatory drugs after resuming an oral diet.

\section{Outcomes}

The primary outcome was superficial SSI, defined using the Center for Disease Control (CDC) criteria, ${ }^{15}$ occurring within 30 days, only skin/subcutaneous tissue involvement, and 1 of the following: purulent drainage, organism isolated from fluid or tissue, or at least 1 of the following signs/symptoms: pain or tenderness, localized swelling, redness, or heat; and the superficial incision was deliberately opened by surgeon without a positive culture. Superficial SSI was assessed by the responsible physician before discharge, and at 1 week and 1 month follow-up. If patients did not visit the outpatient clinic, a standardized telephone interview was used to ascertain wound swelling, pain, discharge, and any patient visits to other hospital/physicians.

Secondary outcomes were postoperative pain, QoL, LOS, and treatment costs. Pain was measured using a 0 to 100 visual analog scale (VAS) at day 1 and 3. Cumulative dosage of opioids was also recorded. QoL was measured at day 3 and 1 month using EuroQol5 and converted to Thai utility scores. ${ }^{16}$ Additional direct medical costs from DPC (LOS, dressing, and re-suture) were estimated from Thailand's standard cost. ${ }^{17}$ Both direct nonmedical (ie, informal care, transportation) and indirect treatment costs (income lost) were obtained by interviewing using standard forms. Informal care costs also included work time lost by caregivers. Transportation cost was captured for patients/ caregivers spent travelling to and from hospital. Thailand's minimum wage of $300 \mathrm{Baht} / \mathrm{d}$ was used for calculation (http://www.mol.go.th/anonymouse/home).

\section{Sample Size}

The pooled superficial SSI rate in DPC was $29.5 \%[95 \%$ confidence interval (CI) $14.8 \%, 44.2 \%$ ] based on 3 previous RCTs. ${ }^{18-20}$ The type I error, power, and ratio were set at 0.05 (2sided), 0.80, and 1:1, respectively. A sample size was estimated based on a difference of $\pm 10 \%$, suggesting a total of 570 patients (285 per group) were needed. Taking into account loss to follow-up of $5 \%$, 600 patients were set as the target.

\section{Statistical Analysis}

The primary analysis was performed by intention to treat (ITT) as for our prespecified analysis plans. ${ }^{21}$ All patients were analyzed according to the groups to which they were originally randomized, accounting for loss to follow-up (modified ITT). ${ }^{22}$ Three additional post hoc analyses were also performed. ${ }^{22,23}$ First, a per-protocol (PP) analysis included only patients who actually received and completed the randomly assigned interventions. Second, as-treated (AT) analysis included patients according to the intervention actually received. Finally, a counterfactual approach using instrumental variable (IV) analysis assessed what outcome would have been seen (or potential outcome) for those patients who $\mathrm{did} / \mathrm{did}$ not comply with the assigned intervention.

Baseline characteristics of the patients are described by intervention groups. Binary regression was used for comparing superficial SSI between groups. SSI incidence, estimated risk difference (RD), and risk ratio (RR), along with $95 \%$ CI, were estimated. Linear or quartile regression analysis was applied to compare LOS, and return to normal activities/work between PC and DPC groups, where appropriate. For pain and QoL scores, a mixed-effect regression model was used to compare mean difference (MD) between groups. The analysis of pain was adjusted for the total opioid dose.

For the counterfactual approach, IV regression was applied considering the assigned intervention and that actually received as the IV and endogenous variable, respectively. Bivariate probit and 2-stage least square regression were applied for SSI and continuous outcomes, respectively. The IV model was adjusted for covariates (eg, age, sex, BMI, smoking, American Society of Anesthesiologists (ASA) classification, diabetes, hypertension, onset time, type of appendicitis, white blood cell count, body temperature, and use of drain).

A planned interim analysis was canceled by consensus of the steering committee, due to the lack of severe adverse events. ${ }^{24}$ Finally, missing data were imputed using a simulation-based approach, ${ }^{25,26}$ as detailed in Appendix I. All analyses were done using STATA version 14.0. $P$ value of less than 0.05 was considered as statistically significant.

\section{RESULTS}

In all, 607 patients (ie, 126, 92, 117, 30, 170, and 72 from Thammasat University, Ramathibodi, Chonburi, Pathum tani, Lampang, and Surin hospitals, respectively) were randomly allocated to DPC $(n=304)$ and PC $(n=303)$ (see Fig. 1). Of these, $148(24 \%)$ had gangrenous appendicitis, and 459 (76\%) had a ruptured appendix, of which $7.1 \%$ ruptured intraoperatively. In the DPC group, the 


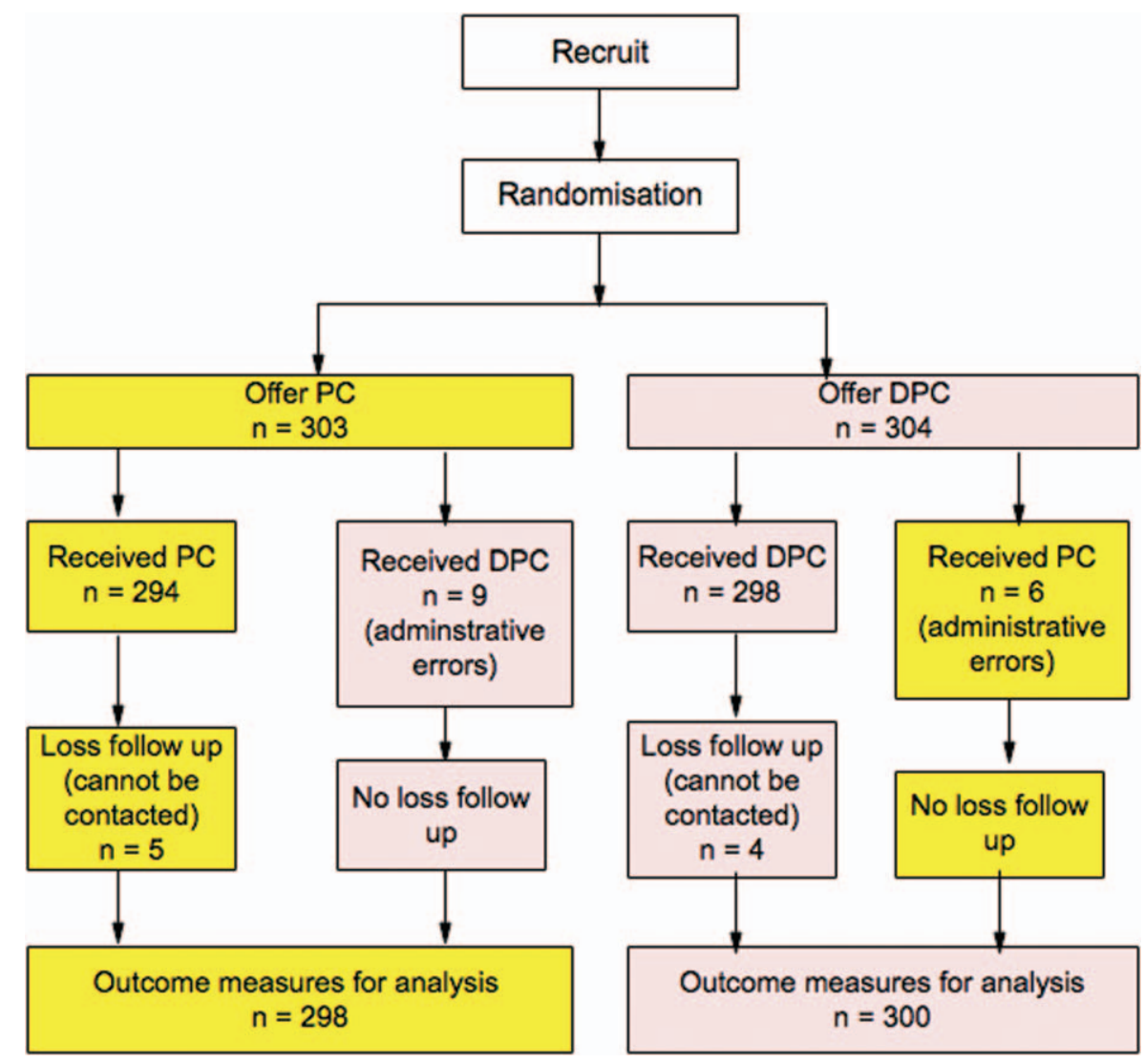

FIGURE 1. CONSORT flow diagram. CONSORT indicates Consolidated Standards of Reporting Trials. statistical significance. A sensitivity analysis of best (no infection) and worst (infection) case scenarios yielded estimated RDs of $-2.6 \%(-7.1 \%, 1.8 \%)$ and $-2.2 \%(-7.1 \%, 2.5 \%)$. Results of PP, AT, and IV analyses were similar, with RDs of $-1.9(-6.5 \%$, $2.6 \%),-1.1 \%(-5.6 \%, 3.4 \%)$, and $-2.8 \%(-7.6 \%, 1.9 \%)$, respectively. The adjusted IV analysis yielded a RD of $-3.6 \%(-8.3 \%$, $1.1 \%$ ). Analyses for all approaches were repeated after imputation (see supplement Table 1, http://links.lww.com/SLA/B308). Results of imputed and unimputed data were very similar for all approaches except for the adjusted IV regression, where imputed data showed a lower effect of PC versus DPV than unimputed data [ie, $-2.6 \%$ $(-7.3 \%, 2.1 \%)$ vs $-3.6 \%(-8.3 \%, 1.1 \%)]$. Combining these RCT data with previous meta-analysis data ${ }^{2}$ yielded a RD of $-1.7 \%$ $(-14.2 \%, 10.8 \%)$.

\section{LOS and Recovery Times}

The ITT analysis indicated the mean LOS was not different, that is, 4.4 days $(4.1,4.6)$ and 4.3 days $(4.0,4.6)$ in the DPC and PC groups, respectively (see Table 3). DPC patients returned to normal activities/work at 3.8 days $(3.4,4.3)$ and 9.0 days $(7.5,10.1)$, compared with 3.6 days $(3.2,4.1)$ and 7.7 days $(6.2,9.1)$ days in the PC patients. Analyses using other approaches (with and without imputation) were again very similar (see Supplement Table 2 and Table 3, http://links.lww.com/SLA/B308).

\section{Postoperative Pain and QoL}

The ITT approach indicated the mean postoperative pain scores were not different, that is, $61(59,63)$ and $62(59,64)$ for DPC and PC groups at day 1 , and $29(27,31)$ for both groups at day 3. The MD was $0.3(-2.5,3.0)$, which was nonsignificant. 


\begin{tabular}{|c|c|c|}
\hline Characteristics & DPC $(n=304)$ & $\mathbf{P C}(\mathbf{n}=303)$ \\
\hline Age, year, mean (SD) & $46(18.0)$ & $45(18.1)$ \\
\hline \multicolumn{3}{|l|}{ Sex, number $(\%)$} \\
\hline Male & $155(51)$ & $169(56)$ \\
\hline Female & $149(49)$ & $134(44)$ \\
\hline BMI, $\mathrm{kg} / \mathrm{m}^{2}$, mean $(\mathrm{SD})$ & $23.4(4.31)$ & $23.4(4.34)$ \\
\hline Smoking, number (\%) & $45(15)$ & $51(17)$ \\
\hline \multicolumn{3}{|l|}{ ASA classification, number $(\%)$} \\
\hline Class I + II & $266(89)$ & $257(85)$ \\
\hline Class III + IV & $34(11)$ & $44(15)$ \\
\hline Diabetes, number $(\%)$ & $31(10.3)$ & $20(6.7)$ \\
\hline Hypertension, number (\%) & $55(18.2)$ & $60(20)$ \\
\hline Symptom onset, h, mean (SD) & $24(15,18)$ & $24(14,18)$ \\
\hline $\begin{array}{l}\text { White blood cell count, cell } / \mathrm{mm}^{3} \text {, } \\
\text { mean (SD) }\end{array}$ & $15561(4965)$ & $15790(4979)$ \\
\hline Body temperature, ${ }^{\circ} \mathrm{C}$, mean $(\mathrm{SD})$ & $37.7(1.0)$ & $37.7(1.1)$ \\
\hline \multicolumn{3}{|l|}{ Fever, number, \% } \\
\hline$\geq 37.8^{\circ} \mathrm{C}$ & $142(47)$ & $148(49)$ \\
\hline$<37.8^{\circ} \mathrm{C}$ & $159(53)$ & $154(51)$ \\
\hline Preoperative utility, median (IQR) & $0.68(0.34,0.80)$ & $0.68(0.34,0.80$ \\
\hline Operative time, min, median (IQR) & $47(14,74)$ & $51(18,78)$ \\
\hline \multicolumn{3}{|l|}{$\begin{array}{l}\text { Operative time classification, } \\
\text { number }(\%)\end{array}$} \\
\hline$\leq 75$ percentile & $232(77)$ & $222(74)$ \\
\hline$>75$ percentile & $68(23)$ & $80(26)$ \\
\hline Used of drain, number (\%) & $62(20.6)$ & $58(19.2)$ \\
\hline \multicolumn{3}{|l|}{ Severity, number (\%) } \\
\hline Gangrene & $76(25)$ & $72(24)$ \\
\hline Ruptured & $228(75)$ & $231(76)$ \\
\hline Intraoperative rupture & $23(7.6)$ & $20(6.6)$ \\
\hline \multicolumn{3}{|c|}{ Visible wound contamination, number (\%) } \\
\hline Exudative fluid & $81(27)$ & $87(29)$ \\
\hline Plus & $118(39)$ & $108(36)$ \\
\hline Feculent material & $38(13)$ & $38(13)$ \\
\hline
\end{tabular}

ASA indicates American Society of Anesthesiologists; IQR, interquartile range; SD, standard deviation.

The QoL scores for PC and DPC groups were quite low at day 3 , that is, $0.54(0.52,0.56)$ and $0.53(0.51,0.55)$, respectively (see Table 3). The scores at day 30 for these corresponding groups increased to $0.79(0.77,0.81)$ and $0.78(0.77,0.80)$, respectively. The overall MD was $0.02(-0.01,0.04)$, which was nonsignificant. Results of other approaches (with and without imputation) were again very similar (see supplement Tables 4 and Table 5, http:// links.lww.com/SLA/B308).

\section{Costs of Treatment}

Our results indicated similar LOS between groups. Direct medical costs were estimated, comprised mainly of the cost of dressings (189 Baht/time, 2 times/d) and re-suture (472 Baht). ${ }^{17}$ As a result, median total direct medical costs were, respectively, $3033(2733,3333)$ and $1200(900,1500)$ for DPC and PC groups, a median difference of $1833(-1884,-1781)$ Baht in favor of PC. Other direct nonmedical costs including informal care and transportation and indirect costs of income lost were not significantly different. However, total costs accounting for both direct and indirect costs were significantly higher for DPC than PC, with a median difference of -2083 Baht $(-2756,-1410)$.

\section{DISCUSSION}

This RCT compared superficial SSI rates between PC and DPC. Our primary results, based on an ITT approach, demonstrated that the superficial SSI rate was $2.7 \%$ lower in PC than DPC, although this was not significant. Although LOS, recovery time, postoperative pain, and QoL were not significantly different, total costs were about 2083 Baht ( 60 US\$) lower in the PC than DPC groups. Although this may appear small (\$60 USD and 56 Euros), this represents about 1 week's wages in Thailand.

Four approaches were applied to test the robustness of the results, that is, modified ITT with/without noninferiority test, PP, AT, and a counterfactual method. The ITT analysis is seen as the least biased because it preserves the original random allocation as recommended in the Consolidated Standards of Reporting Trials guideline. ${ }^{27}$ However, the ITT estimate may be biased if there is protocol violation and loss to follow-up as in our study. The estimated RD was $-2.7 \%$, which may be biased away from the null because protocol violations were higher in the PC than in the DPC groups, that is, $4.6 \%$ versus $3.3 \%$. The PP and AT analyses may be more relevant than the ITT analysis in assessing the actual effects of interventions received. The PP analysis considers only patients who were randomly allocated and complied with their allocation, whereas the AT analysis considers actual intervention received, regardless of randomization. ${ }^{28}$ The PP analysis is prone to selection bias because the randomization is broken due to nonadherence, whereas the AT approach deals with data as if it was observational. Therefore, both approaches are potentially biased if the pattern of protocol violation and confounders are different between the 2 groups. The IV regression is applied to estimate what the intervention effect would have been (ie, counterfactual effects) if patients who were randomly assigned to PC actually received DPC, or vice versa. ${ }^{29,30}$ The IV regression itself can adjust for observed and unobserved confounders. As a result, the RD between PC versus DPC groups was $-2.8 \%$, which was about $0.1 \%$ higher than the ITT estimate. Surprisingly, the IV regression with adjustment for covariates yielded a higher effect of PC than the IV regression without adjustment, with a RD of $-3.6 \%$. Missing data for some covariates used in the adjusted model might have played a role in this discrepancy, given the RDs of the 2 IV models were closer to each other after applying multiple imputations to fill in missing data. Analyses for all approaches using complete/unimputed and imputed data showed similar directions of intervention effect in with favor of PC, although none reached statistical significance for superiority.

Our results confirm findings of the previous systematic review and meta-analysis, ${ }^{2}$ which demonstrated similarly lower superficial SSI in PC than in DPC groups, that is, $23 \%(12 \%, 33 \%)$ versus $26 \%$ $(10 \%, 42 \%)$, respectively. However, the estimated incidence from this meta-analysis was much higher than our RCT, which might be explained by the fact that a few included studies did not prescribe antibiotic prophylaxis and thus had superficial SSI rates as high as $37 \%{ }^{31}$ and $57 \% .^{19}$ Our study was planned to show superiority; however, it could also be interpreted as a noninferiority trial. Recommendations are that a noninferiority margin should be set to not exceed $25 \%$ of the standard effect. ${ }^{23,32,33}$ An estimate of $20 \%$ (ie, $10 \%$ of SSI in DPC) would give a noninferiority margin of $2 \%$, and therefore our observed RD of $-2.7 \%$ (upper end of CI $1.9 \%$ ) would have met this noninferiority test $(Z=-2.02, \mathrm{SE}=0.023, P=0.021)$.

Our results are contrary to the RCT by Duttaroy et al, ${ }^{9}$ which demonstrated much higher superficial SSI in PC than DPC (ie, $45.2 \%$ vs $2.7 \%$ ). This might be because they considered not only ruptured or gangrenous appendicitis but also peptic and typhoid perforations. In addition, the incision was midline, whereas ours was a right lowerquadrant incision, which is shorter and hence less prone to infection. Five per cent of patients in that study died, reflecting the greater severity of patients.

We did not find a difference in LOS between PC and DPC, which was in contrast to the previous systematic review and metaanalysis $^{2}$ that found longer LOS in DPC than PC. This might be explained by the fact that patients in both groups were routinely prescribed preoperative intravenous antibiotics, and these were 

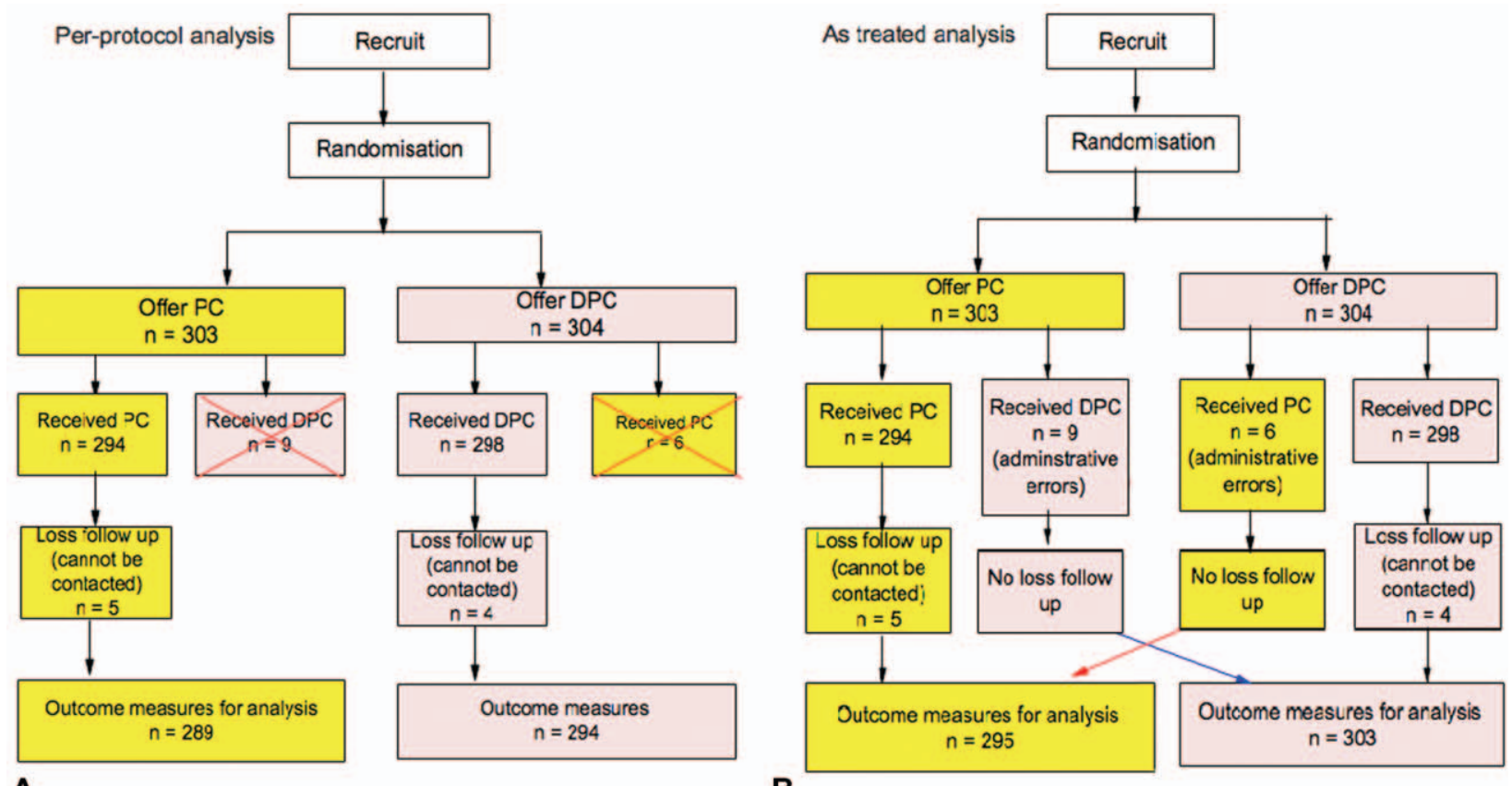

A

B

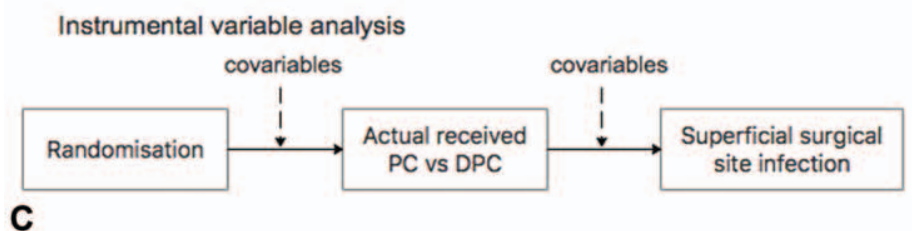

FIGURE 2. Different approaches analysis.

continued until body temperature $<37.8^{\circ} \mathrm{C}$ for 24 to 48 hours after operation, hence prolonging LOS in the PC group.

In all, 476 (78\%) patients had intra-abdominal fluid cultures; $105(22 \%)$ were positive with hospital-acquired organisms including 47\% P aeruginosa, 45\% Escherichia coli (ESBL), 8\% multidrugresistant $E$ coli, and 1\% Acinetobacter bauminii. Among 52 superficial SSIs, $26(50 \%)$ had wound cultures after opening the wound, and $2 / 3$ were gram-negative positive bacteria. Of these, 5 (29\%) were resistant strains (ie, 2 P aeruginosa, 2 multidrug-resistant $E$ coli, and
1 ESBL); resistant organisms could have been seeded during wound care or have developed during hospitalization. ${ }^{34}$

Our study cannot inform the use of other types of appendectomy (eg, laparoscopic). Laparoscopic appendectomy can significantly reduce the rate of superficial SSI in complicated appendicitis as compared with open procedure ${ }^{35}$ but this option is not always available, sometimes due to technical reasons and sometimes due to cost constraints. Our study also cannot inform the use of other types of DPC (eg, subcutaneous drain ${ }^{36,37}$ and wound wicks ${ }^{38}$ ) in which

TABLE 2. Comparison of Superficial SSI Rates Between PC and DPC Using Different Approaches

\begin{tabular}{lcccr}
\hline Approach & DPC & PC & RR (95\% CI) & RD (95\% CI) \\
\hline ITT & 300 & 298 & $0.74(0.44,1.25)$ & $-0.027(-0.071,0.019)$ \\
$\quad$ Rate $(\%, 95 \%$ CI) & $10(6.6,13.3)$ & $7.3(4.4,10.3)$ & & 0.258 \\
PP & 294 & 289 & $0.80(0.47,1.36)$ & $-0.019(-0.065,0.026)$ \\
$\quad$ n & $9.5(6.2,12.9)$ & $7.6(4.6,10.7)$ & & 0.411 \\
$\quad$ Rate $(\%, 95 \%$ CI) & 303 & 295 & $0.88(0.52,1.48)$ & $-0.011(-0.56,0.034)$ \\
AT & $9.2(5.9,12,5)$ & $8.1(5.0,11.3)$ & & 0.632 \\
$\quad$ n & 294 & 293 & $0.72(0.32,1.12)$ & $-0.027(-0.076,0.019)$ \\
$\quad$ Rate $(\%, 95 \%$ CI) & $10.1(6.6,13.8)$ & $7.4(4.4,10.3)$ & & $-0.036(-0.083,0.011)$ \\
$\quad$ n & 276 & 282 & $0.66(0.30,1.01)$ & 0.243 \\
$\quad$ Rate $(\%, 95 \%$ CI) & $10.7(7.0,14.1)$ & $6.9(4.1,9.8)$ & & 0.118 \\
IV (with adjusted variables) & & & \\
$\quad$ Rate $(\%, 95 \%$ CI) & & & \\
\hline
\end{tabular}


TABLE 3. Comparisons of Length of Stay, Recovery Times, Postoperative Pain and Costs of Treatment Between PC and DPC Groups Based on ITT Approach

\begin{tabular}{|c|c|c|c|c|c|}
\hline \multirow[b]{2}{*}{ Outcomes } & \multicolumn{2}{|r|}{ DPC } & \multicolumn{2}{|r|}{ PC } & \multirow[b]{2}{*}{ MD $(95 \%$ CI $)$} \\
\hline & $\mathbf{n}$ & $\bar{X}(95 \%$ CI $)$ & $\mathbf{n}$ & $\bar{X}(95 \%$ CI $)$ & \\
\hline Length of stay, day & 304 & $4.4(4.1,4.6)$ & 302 & $4.3(4.0,4.6)$ & $-0.1(-0.5,0.3)$ \\
\hline Return to normal activities, day & 271 & $3.8(3.4,4.3)$ & 273 & $3.6(3.2,4.1)$ & $-0.2(-0.8,0.4)$ \\
\hline Return to work, day & 265 & $9.0(7.5,10.1)$ & 267 & $7.7(6.2,9.1)$ & $-1.3(-3.4,0.8)$ \\
\hline \multicolumn{6}{|l|}{ Postoperative pain, VAS } \\
\hline Day 1 & 301 & $61(59,63)$ & 299 & $62(59,64)$ & $0.3(-2.5,3.0)$ \\
\hline Day 3 & 292 & $29(27,31)$ & 295 & $29(27,31)$ & \\
\hline \multicolumn{6}{|l|}{ QoL, utility scores } \\
\hline Day 3 & 300 & $0.53(0.51,0.55)$ & 299 & $0.54(0.52,0.56)$ & $0.02(-0.01,0.04)$ \\
\hline \multirow[t]{2}{*}{ Day 30} & 289 & $0.78(0.77,0.80)$ & 287 & $0.79(0.77,0.81)$ & \\
\hline & $\mathrm{N}$ & Median (IQR) & $\mathrm{N}$ & Median (IQR) & Median difference $(95 \% \mathrm{CI})$ \\
\hline \multicolumn{6}{|l|}{ Costs of treatment, Baht* } \\
\hline Added direct medical costs ${ }^{\dagger}$ & 304 & $3033(2733,3333)$ & 302 & $1200(900,1500)$ & $-1833(-1884,-1781)$ \\
\hline \multicolumn{6}{|l|}{ Direct nonmedical costs } \\
\hline Informal care (during admission) & 274 & $1050(600,1800)$ & 271 & $1050(600,1800)$ & $0(-133,133)$ \\
\hline Informal care (during recovery) & 272 & $450(0,1650)$ & 272 & $300(0,1200)$ & $-150(-440,140)$ \\
\hline Transportation & 274 & $300(100,800)$ & 271 & $300(100,600)$ & $0(-80,80)$ \\
\hline \multicolumn{6}{|l|}{ Indirect costs } \\
\hline Income lost $\mathrm{t}^{\ddagger}$ & 265 & $2100(600,3000)$ & 267 & $2100(900,3000)$ & $0(-266,266)$ \\
\hline Total costs & 248 & $6398(4343,8558)$ & 250 & $4305(2750,6100)$ & $-2083(-2756,-1410)$ \\
\hline
\end{tabular}

${ }^{*}$ Analysis using quantile regression analysis.

$\dagger$ Added direct medical costs from DPC were 1833 Baht (1361 Baht for dressing changes [189 Baht/times, 2 times/d for 3.6 days], and 472 Baht for re-suture).

$\ddagger$ Minimum wages of $300 \mathrm{Baht} / \mathrm{d}$ in Thailand was used.

re-suturing is not required, thus potentially reducing the cost difference. Our findings may also be generalizable to other operations that require laparotomy and where DPC might be considered, but this would need to be specifically addressed.

Our study also indicated that DPC costs were about 2083 Baht higher than PC, without a benefit over PC in terms of efficacy and QoL. Therefore, PC was more cost-effective that DPC. Given a midyear population in Thailand 2015 of 65,729,098 (http://stat.dopa.go.th/stat/statnew/upstat_age_disp.php), an estimated rate of appendectomy of $14 / 10,000 / \mathrm{yr}^{1}$ would result in 92,020 appendectomies/yr in Thailand. The prevalence of complicated appendicitis from our study was $18.2 \%,{ }^{39}$ indicating 16,748 cases of complicated appendicitis across the country. Using PC routinely instead of DPC in all patients would save about 34,886,084 Baht country-wide.

To the best of our knowledge, our study is the largest RCT to date to assess the efficacy of DPC and PC in adult complicated appendicitis. Patients were randomized to receive DPC or PC to balance unknown and known risk factors (eg, BMI, diabetes, ASA classification, operative time, and degree of contamination, etc $)^{40-43}$ of superficial SSI. After adjusting for wound management (ie, PC vs DPC), we found that diabetes, operative duration, visible feculent contamination, and ruptured appendicitis were risk factors for SSI, but BMI was not (data not shown). Although some protocol violations occurred, we applied modified ITT, PP, AT, and counterfactual IV regression to deal with these, and results were all similar and consistent. In addition, multiple imputation was applied to fill in missing data, and results were again consistent with un-imputed data. Although we could not blind the outcome assessors who monitored occurrence of superficial SSI, we tried to reduce any ascertainment bias by using standard protocols for diagnosing superficial SSI.

\section{CONCLUSIONS}

Primary wound closure is not different to DPC in adults with complicated appendicitis (ie, gangrenous and ruptured) with respect to SSI or LOS, although there are lower costs.

\section{ACKNOWLEDGMENTS}

We would like to thank all involving persons that provide us kindly, intentionally help in many ways with moral support. These included every assistant in all collaborating centers and at our DMU, Section for Clinical Epidemiology and Biostatistics, Faculty of Medicine, Ramathibodi Hospital.

\section{REFERENCES}

1. Lee JH, Park YS, Choi JS. The epidemiology of appendicitis and appendectomy in South Korea: national registry data. J Epidemiol. 2010;20:97-105.

2. Siribumrungwong B, Noorit P, Wilasrusmee C, et al. A systematic review and meta-analysis of randomised controlled trials of delayed primary wound closure in contaminated abdominal wounds. World J Emerg Surg. 2014;9:49.

3. St Peter SD, Sharp SW, Holcomb GW 3rd, et al. An evidence-based definition for perforated appendicitis derived from a prospective randomized trial. J Pediatr Surg. 2008;43:2242-2245.

4. Urban JA. Cost analysis of surgical site infections. Surg Infect (Larchmt). 2006;7(Suppl 1):S19-S22.

5. Hepburn HH. Delayed primary suture of wounds. Br Med J. 1919;1:181-183.

6. Fogdestam I, Jensen FT, Nilsson SK. Delayed primary closure. Bloodflow in healing rat skin incisions. Scand J Plast Reconstr Surg. 1981;15: $81-85$.

7. Fogdestam I, Niinikoski J. Delayed primary closure. Tissue gas tensions in healing rat skin incisions. Scand J Plast Reconstr Surg. 1981;15:9-14.

8. Fogdestam I. A biomechanical study of healing rat skin incisions after delayed primary closure. Surg Gynecol Obstet. 1981;153:191-199.

9. Duttaroy DD, Jitendra J, Duttaroy B, et al. Management strategy for dirty abdominal incisions: primary or delayed primary closure? A randomized trial. Surg Infect (Larchmt). 2009;10:129-136.

10. Russell GG, Henderson R, Arnett G. Primary or delayed closure for open tibial fractures. J Bone Joint Surg Br. 1990;72:125-128.

11. Brasel KJ, Borgstrom DC, Weigelt JA. Cost-utility analysis of contaminated appendectomy wounds. J Am Coll Surg. 1997;184:23-30.

12. Leaper DJ, Edmiston CE. World Health Organization: global guidelines for the prevention of surgical site infection. J Hosp Infect. 2017;95: $135-136$.

13. Siribumrungwong B, Srikuea K, Thakkinstian A. Comparison of superficial surgical site infection between delayed primary and primary wound closures in ruptured appendicitis. Asian J Surg. 2014;37:120-124. 
14. Solomkin JS, Mazuski JE, Bradley JS, et al. Diagnosis and management of complicated intra-abdominal infection in adults and children: guidelines by the Surgical Infection Society and the Infectious Diseases Society of America. Clin Infect Dis. 2010;50:133-164.

15. Horan TC, Andrus M, Dudeck MA. CDC/NHSN surveillance definition of health care-associated infection and criteria for specific types of infections in the acute care setting. Am J Infect Control. 2008;36:309-332.

16. Tongsiri S, Cairns J. Estimating population-based values for EQ-5D health states in Thailand. Value Health. 2011;14:1142-1145.

17. Riewpaiboon A. Standard cost lists for health economic evaluation in Thailand. J Med Assoc Thai. 2014;97(Suppl 5):S127-S134.

18. Chatwiriyacharoen W. Surgical wound infection post surgery in perforated appendicitis in children. J Med Assoc Thai. 2002;85:572-576.

19. Cohn SM, Giannotti G, Ong AW, et al. Prospective randomized trial of two wound management strategies for dirty abdominal wounds. Ann Surg. 2001;233:409-413.

20. Khammash MR, Ayyash K. Wound infection in primary versus delayed primary wound closure in cases of perforated and gangrenous appendicitis. Saudi Med J. 1994;15:408-410.

21. Fisher LD, Dixon DO, Herson J, et al. Intention to treat in clinical trials. In: Peace KE, editor. Statistical Issues in Drug Research and Development. New York: Marcel Dekker; 1990:331-350.

22. McNamee R. Intention to treat, per protocol, as treated and instrumental variable estimators given non-compliance and effect heterogeneity. Stat Med. 2009;28:2639-2652.

23. Gao P, Ware JH. Assessing non-inferiority: a combination approach. Stat Med. 2008;27:392-406.

24. Trotta F, Apolone G, Garattini S, et al. Stopping a trial early in oncology: for patients or for industry? Ann Oncol. 2008;19:1347-1353.

25. Rubin DB, Schenker N. Multiple imputation in health-care databases: an overview and some applications. Stat Med. 1991;10:585-598.

26. White IR, Royston P, Wood AM. Multiple imputation using chained equations: issues and guidance for practice. Stat Med. 2011;30:377-399.

27. Altman DG, Schulz KF, Moher D, et al. The revised CONSORT statement for reporting randomized trials: explanation and elaboration. Ann Intern Med. 2001;134:663-694.

28. Hernan MA, Hernandez-Diaz S. Beyond the intention-to-treat in comparative effectiveness research. Clin Trials. 2012;9:48-55.

29. Shrier I, Steele RJ, Verhagen E, et al. Beyond intention to treat: what is the right question? Clin Trials. 2014;11:28-37.
30. Dunn G, Emsley R, Liu H, et al. Evaluation and validation of social and psychological markers in randomised trials of complex interventions in mental health: a methodological research programme. Health Technol Assess. 2015;19:1-115 (v-vi).

31. Pettigrew RA. Delayed primary wound closure in gangrenous and perforated appendicitis. Br J Surg. 1981;68:635-638.

32. Julious SA. Sample sizes for clinical trials with normal data. Stat Med. 2004;23:1921-1986.

33. D'Agostino RB Sr, Massaro JM, Sullivan LM. Non-inferiority trials: design concepts and issues: the encounters of academic consultants in statistics. Stat Med. 2003;22:169-186.

34. Stone HH, Hester TR Jr. Topical antibiotic and delayed primary closure in the management of contaminated surgical incisions. J Surg Res. 1972;12:70-76.

35. Yu MC, Feng YJ, Wang W, et al. Is laparoscopic appendectomy feasible for complicated appendicitis? A systematic review and meta-analysis. Int J Surg. 2017;40:187-197.

36. Imada $\mathrm{S}$, Noura $\mathrm{S}$, Ohue $\mathrm{M}$, et al. Efficacy of subcutaneous penrose drains for surgical site infections in colorectal surgery. World J Gastrointest Surg. 2013;5:110-114.

37. Arer IM, Yabanoglu H, Aytac HO, et al. The effect of subcutaneous suction drains on surgical site infection in open abdominal surgery A prospective randomized study. Ann Ital Chir. 2016;87:49-55.

38. McGreal GT, Joy A, Manning B, et al. Antiseptic wick: does it reduce the incidence of wound infection following appendectomy? World J Surg. 2002;26:631-634.

39. Chumpon Wilasrusmee BS, Phuwapraisirisan S, Poprom N, et al. Developing and validating of Ramathibodi Appendicitis Score (RAMA-AS) for diagnosis of appendicitis in suspected appendicitis patients. 2017 (submitted).

40. Lawson EH, Hall BL, Ko CY. Risk factors for superficial vs deep/organ-space surgical site infections: implications for quality improvement initiatives. JAMA Surg. 2013;148:849-858.

41. National Nosocomial Infections Surveillance (NNIS) System Report, data summary from January 1992 through June 2004, issued October 2004. Am J Infect Control. 2004;32:470-485.

42. Korol E, Johnston K, Waser N, et al. A systematic review of risk factors associated with surgical site infections among surgical patients. PLoS One. 2013;8:e83743.

43. Thelwall S, Harrington P, Sheridan E, et al. Impact of obesity on the risk of wound infection following surgery: results from a nationwide prospective multicentre cohort study in England. Clin Microbiol Infect. 2015;21:1008.e1-1008.e8. 